\title{
OSCILLATOR STRENGTHS OF NEUTRAL AND SINGLY IONISED MOLYBDENUM
}

\author{
S. E. Schnehage, K. Danzmann†, R. Künnemeyer, and M. Kock \\ Institut für Plasmaphysik, Universität Hannover, Callinstr. 38, 3000 Hannover, West Germany
}

(Received 30 September 1982)

\begin{abstract}
Oscillator strengths of $174 \mathrm{Mo}$ (I) and $58 \mathrm{Mo}$ (II) lines in the range $2470-5570 \AA$ were obtained from wall-stabilised arc and hollow cathode measurements. Sets of relative $f$-values were determined by a combination of hook and emission measurements requiring no assumptions concerning the plasma state. The sets have been enlarged considerably by evaluating spectra recorded with a Fourier spectrometer. The relative $\operatorname{Mo}(\mathrm{I}) f$-values were converted to an absolute scale by means of radiative lifetimes measured by $\mathbf{P}$. Zimmermann and his group. The overall uncertainties are within $10-35 \%$. Since lifetimes of $\mathrm{Mo}$ (II) levels are not yet available, only relative Mo(II) $f$-values are presented.
\end{abstract}

\section{INTRODUCTION}

In astrophysics and for fusion research, reliable oscillator strengths are needed, especially for the heavy elements. A method frequently used to obtain large sets of $f$-values for lines of different excitation potentials is that based upon emission spectroscopy on suitable plasma light sources such as cascaded arcs. ' A more promising method, which reduces the number of assumptions required, is the combination of hook and emission measurements. ${ }^{26}$ Thus, large sets of relative $f$-values can be determined without any assumptions concerning the plasma state. The absolute calibration of the relative data is preferably done by means of radiative lifetimes.

The objective of the present investigation was the determination of reliable oscillator strengths of neutral and singly ionised molybdenum.

\section{EXPERIMENTAL PROCEDURE}

The hook measurements were performed with a three chamber cascaded arc, which consisted of a stack of brass plates, each $10 \mathrm{~mm}$ thick with a central bore of $16 \mathrm{~mm}$. The plates were electrically insulated from one another by $1 \mathrm{~mm}$ thick Pertinax sheets. The plates were cooled axially by forced water cooling. The arc length was $120 \mathrm{~mm}$ between a ring anode and two tapered cathodes, all made of tungsten. The arc was operated in argon at atmospheric pressure and at a current of $40 \mathrm{~A}$ supplied by a d.c. generator $(460 \mathrm{~V} / 120 \mathrm{~A})$.

For the excitation of molybdenum in the arc, we used an admixture of molybdenum pentachloride $\left(\mathrm{MoCl}_{5}\right.$ : b.p. $\left.268^{\circ} \mathrm{C}\right)$. The vapor was generated in a small ceramic oven attached to the arc chamber.

The set-up for our hook experiment was similar to that described in an earlier paper. ${ }^{3}$ It consisted of the cascaded arc, a high-power capillary discharge as a continuum light source, a Michelson interferometer with quartz optics, and a stigmatic spectrograph. The spectrograph was a $3.4 \mathrm{~m}$ Ebert configuration with a plane grating of 1200 lines $\mathrm{mm}^{-1}$ producing a reciprocal linear dispersion of $2.4 \AA \mathrm{mm}^{-1}$ in the first order. The spectra were photographed on Ilford FP 4 film. They were evaluated by means of a computer. Only hooks with a separation of at least $300 \mathrm{~m} \AA$ were evaluated.?

The emission measurements were performed with hollow cathodes. We have used commercially available hollow cathodes (Perkin-Elmer, No. 029287) which we have operated at a current of $30 \mathrm{~m} \AA$. A problem in using this type of hollow cathode is the examination of the optical depth of the plasma layer from which the lines under investigation are emitted. Therefore, we have again used our high current hollow cathode. ${ }^{6}$

†Present address: Stanford University, Department of Physics, Stanford, CA 94305, U.S.A. 
The cathode has a length of $100 \mathrm{~mm}$ and a central bore of $8.5 \mathrm{~mm}$. The discharge was operated either in argon or in neon at a current of $2 \mathrm{~A}$

The spectra were recorded photoelectrically by using a photon counting system and a 1-m McPherson monochromator with a plane grating of 2400 lines $\mathrm{mm}^{-1}$ having a resolution of about $55 \mathrm{~m} \AA$. The line intensities were calibrated with a carbon are as a radiation standard. ${ }^{8.9}$ For the u.v. region below $3300 \AA$, we used a low-current argon arc designed and calibrated by PTB $^{10}$ in Berlin.

The present program was complemented by measurements with the $1-\mathrm{m}$ fourier transform spectrometer at the Kitt Peak National Observatory. Tucson. Arizona. Experimental details such as the calibration of the spectra are described elsewhere." For molybdenum, we obtained one spectrum in the range $3000-5100 \AA$.

All emission lines were tested and shown to be free of self-absorption. For the Perkin-Elmer hollow cathode, we measured relative intensities of lines with the same upper level but of different strengths at various discharge currents. The constancy of the intensity ratio then was a check that the lines were emitted from an optically thin layer

Since the lines emitted from our hollow cathode are Doppler-broadened, we have measured the widths of the lines by means of a highly resolving Fabry-Perot interferometer and estimated the maximum optical thickness by the following relationship:

$$
\tau_{\max } \leq 0.94 J \Delta \lambda_{n} B
$$

where $J$ is the integrated line intensity, $B$ the Kirchhoff-Planck function and $\Delta /$, , the FWHM of the line under investigation. Since only lines with $t_{\text {mal }}<0.1$ have been evaluated. no corrections were necessary for an optically thin layer.

\section{RESULTS AND DISCUSSION}

For the $174 \mathrm{Mo}(\mathrm{I})$ lines investigated, we have obtained three independent sets of relative $f$-values from a combination of hook measurements for lines of common lower levels, with emission measurements for lines originating from common upper levels. In the same manner, we obtained one set of relative $f$-values for the 58 Mo(II) lines.

For an absolute calibration of the Mo(I) sets, we used lifetimes which have been measured by P. Zimmermann ${ }^{12}$ and his group (see Table 1). The lifetime of the $2^{7} P_{4}{ }^{11}$ level has been published by these authors ${ }^{1.3}$ together with additional Mo(l) lifetimes belonging to the septet system. We have calculated absolute $f$-values from the first three lifetimes of Table 1 and our measured branching ratios. The final data comprising $174 \mathrm{Mo}(\mathrm{I})$ lines are listed in Table 2.

The accuracy of the $f$-values was estimated to be within $10-35^{\prime \prime}$ "because of errors in the emission and hook measurements (the standard deviation of the mean was evaluated from up to seven independent measurements) and the uncertainty of the lifetime

The completeness of the branching ratios has been checked by means of the Fourier spectrum. For the level $y^{5} F_{1}{ }^{0}$, only the lines at 6812 and $6787 \AA$ could not be measured. We have taken into account this contribution $\left(1^{\prime \prime}{ }_{0}\right)$ by using the calculated $f$-values given by Kurucz and Peytreman. ${ }^{14}$

Table 1. Mo(I) lifetimes measured by P. Zimmermann and his group. " " The uncertainty is 5 " "."

\begin{tabular}{|c|c|c|}
\hline Level 1 & 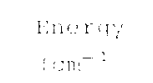 & 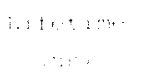 \\
\hline $\mathrm{z}^{7} \mathrm{P}_{4}^{0}$ & $26 \quad 2 c, \ldots 1 s$ & $\therefore$ \\
\hline$z^{5} P^{\prime}=$ & $208 ; 1, \ldots+$ & \\
\hline$Y^{5} \mathrm{~F}_{1}^{0}$ & $\$ 1011,4$ & $\cdots:$ \\
\hline$y^{5} F_{2}^{5}$ & $41 \quad \because: 2, \ldots$ & \\
\hline
\end{tabular}


Table 2. Absolute oscillator strengths of $\mathrm{Mo}(\mathrm{I})$ lines: $\left({ }^{*}\right)$, determined from a Fourier spectrum; ('), wavelength corrected.

\begin{tabular}{|c|c|c|c|c|}
\hline $\begin{array}{l}\text { Wavelength } \\
\text { (A) }\end{array}$ & Transition & $E_{(\mathrm{m}}$ & $\lg (g f)$ & $\begin{array}{c}\text { Error } \\
\text { (8) }\end{array}$ \\
\hline 2471.95 & $a^{5} S_{2}-w^{5} P_{3}^{0}$ & 51209.92 & -0.10 & 25 \\
\hline 2505.08 & $a^{5} D_{1}-v^{5} P_{2}^{0}$ & 51049.60 & -1.20 & 22 \\
\hline 2514.62 & $a^{5} D_{2}-w^{5} P^{0}$ & $\begin{array}{ll}51 & 209.92\end{array}$ & -0.87 & 25 \\
\hline 2524.80 & $a^{5} D_{2}-v^{5} p_{2}^{0}$ & 51049.60 & -0.48 & 12 \\
\hline 2540.44 & $a^{5} D_{3}-w^{5} P_{3}^{0}$ & $\begin{array}{ll}51 & 209.92\end{array}$ & -0.31 & 19 \\
\hline 2548.23 & $a^{5} S_{2}-v^{5} P_{3}^{0}$ & 49999.49 & -0.21 & 31 \\
\hline 2550.84 & $a^{5} D_{3}-v^{5} P_{2}^{0}$ & 51049.60 & -0.48 & 22 \\
\hline 2567.05 & $a^{5} S_{2}-w^{5} P_{2}^{0}$ & 49.711 .86 & -0.31 & 16 \\
\hline 2572.33 & $a^{5} D_{4}-w^{5} P_{3}^{0}$ & 51209.92 & +0.29 & 24 \\
\hline 2582.15 & $a^{5} s_{2}-w^{5} p_{1}^{0}$ & 49484.14 & -0.39 & 10 \\
\hline 2583.22 & $a^{5} S_{2}-t^{5} F_{3}^{0}$ & 49468.05 & -1.89 & 16 \\
\hline 2591.97 & $a^{5} D_{1}-w^{5} P_{2}^{0}$ & 49711.86 & -0.83 & 12 \\
\hline 2593.59 & $a^{5} D_{2}-v^{5} P_{3}^{0}$ & 49999.49 & -0.45 & 33 \\
\hline 2595.40 & $a^{5} D_{0}-w^{5} P_{1}^{0}$ & 49484.14 & -0.74 & 11 \\
\hline 2607.37 & $a^{5} D_{1}-w^{5} P Q$ & 49484.14 & -0.37 & 10 \\
\hline 2611.21 & $a^{5} D_{4}-w^{3} G^{9}$ & $50 \quad 631.35$ & -0.26 & 21 \\
\hline 2613.09 & $a^{5} D_{2}-w^{5} P_{2}^{0}$ & 49711.86 & -0.18 & 13 \\
\hline 2615.39 & $a^{5} D_{0}-t^{5} F_{1}^{0}$ & $49 \quad 189.71$ & -0.67 & 10 \\
\hline 2616.78 & $a^{5} D_{1}-t^{5} F_{2}$ & 49346.30 & -0.38 & 14 \\
\hline 2621.07 & $a^{5} D_{3}-v^{5} P_{3}^{0}$ & 49999.49 & -0.41 & 31 \\
\hline 2627.55 & $a^{5} D_{1}-t^{5} F ?$ & $49 \quad 189.71$ & -0.55 & 21 \\
\hline 2628.74 & $a^{5} D_{2}-w^{5} P !$ & $49 \quad 484.14$ & -0.37 & 14 \\
\hline 2635.56 & $a^{5} D_{3}-u^{3} F_{3}^{0}$ & 49789.83 & -0.87 & 16 \\
\hline 2638.30 & $a^{5} D_{2}-t^{5} F_{2}^{0}$ & 49346.30 & -0.30 & 14 \\
\hline 2640.99 & $a^{5} D_{3}-w^{5} P_{2}$ & 49711.86 & +0.05 & 16 \\
\hline 2649.46 & $a^{5} D_{3}-t^{5} F_{4}^{0}$ & 49590.83 & +0.24 & 14 \\
\hline 2655.03 & $a^{5} D_{4}-v^{5} p_{3}^{0}$ & 49999.49 & +0.30 & 21 \\
\hline 2658.11 & $a^{5} D_{3}-t^{5} F_{3}^{0}$ & 49468.05 & -0.12 & 19 \\
\hline 2666.74 & $a^{5} D_{3}-t^{5} F_{2}^{0}$ & 49346.30 & -1.37 & 13 \\
\hline 2670.32 & $a^{5} s_{2}-v^{3} D_{3}^{0}$ & $48 \quad 205.95$ & -0.92 & 23 \\
\hline 2679.86 & $a^{5} D_{4}-t^{5} F_{\xi}^{0}$ & 49650.58 & +0.55 & 21 \\
\hline 2681.02 & $a^{5} D_{3}-x^{5} G_{4}^{0}$ & $49 \quad 146.69$ & -0.85 & 27 \\
\hline 2697.80 & $a^{5} D_{0}-u^{5} F_{j}^{0}$ & $48 \quad 022.23$ & -0.94 & 11 \\
\hline 2701.03 & $a^{5} D_{3}-v^{5} D_{4}^{0}$ & $48 \quad 870.44$ & -0.62 & 12 \\
\hline 2701.09 & $a^{5} s_{2}-v^{3} D_{1}^{0}$ & 47779.41 & -0.77 & 12 \\
\hline 2706.11 & $a^{5} D_{1}-v^{3} D_{2}^{0}$ & 48085.26 & -0.95 & 22 \\
\hline 2710.74 & $a^{5} D_{1}-u^{5} F_{1}^{0}$ & $48 \quad 022.23$ & -1.14 & 13 \\
\hline 2720.17 & $a^{5} D_{2}-v^{3} D_{3}^{0}$ & $48 \quad 205.95$ & -0.86 & 10 \\
\hline 2725.15 & $a^{5} D_{1}-u^{5} F_{2}^{0}$ & 47827.22 & -0.82 & 15 \\
\hline 2733.39 & $a^{5} D_{2}-u^{5} F_{3}^{0}$ & $48 \quad 028.22$ & +0.43 & 66 \\
\hline 2733.84 & $a^{5} D_{2}-u^{5} F_{1}^{0}$ & $48 \quad 022.23$ & -0.33 & 17 \\
\hline 2751.47 & $a^{5} D_{3}-u^{5} F_{4}^{0}$ & $48 \quad 192.05$ & -0.31 & 23 \\
\hline
\end{tabular}


Table 2. (Contd)

\begin{tabular}{|c|c|c|c|c|}
\hline $\begin{array}{l}\text { Wavelergth } \\
(\AA)\end{array}$ & Transition & $\begin{array}{l}E_{m} \\
\left(\mathrm{~cm}^{-1}\right)\end{array}$ & $\lg (g f)$ & $\begin{array}{c}\text { Error } \\
\text { (8) }\end{array}$ \\
\hline 2761.53 & $a^{5} D_{4}-U^{5} F_{5}^{0}$ & $48 \quad 547.44$ & -0.25 & 21 \\
\hline 2779.47 & $a{ }^{5} D_{1}-w^{3} D_{2}^{0}$ & $47 \quad 110.24$ & -1.02 & 26 \\
\hline 2787.83 & $a^{5} D_{4}-v^{3} D_{3}^{0}$ & $48 \quad 205.95$ & -0.27 & 21 \\
\hline 2788.91 & $a^{5} D_{4}-u^{5} F_{4}^{0}$ & 48.192 .05 & -0.76 & 21 \\
\hline 2797.93 & $a^{5} D_{2}-w^{3} D_{3}^{0}$ & $47 \quad 184.58$ & -0.50 & 22 \\
\hline 2801.46 & $a^{5} S_{2}-v^{5} F_{3}^{0}$ & $46 \quad 453.42$ & -0.73 & $1^{1}$ \\
\hline 2817.50 & $a^{5} D_{0}-v^{5} F_{i}^{0}$ & $46 \quad 447.97$ & -1.10 & 15 \\
\hline 2905.41 & $a^{5} G_{z}-v^{5} p ?$ & 51049.60 & + o. is & 20 \\
\hline 3038.36 & $a^{5} G_{4}-t^{5} F_{5}^{0}$ & $49 \quad 650.58$ & $-\quad .20$ & 25 \\
\hline 3047.32 & $a^{5} G_{5}-t^{5} F_{4}^{c}$ & 49590.83 & $-\gamma \cdot 1 \cdot \dot{t}$ & 18 \\
\hline $3050.21::$ & $a^{5} G_{3}-t^{5} E_{3}$ & 49468.05 & $-\quad \therefore y$ & 25 \\
\hline $3055.32:$ & $a^{5} G_{4}-t^{5} F_{3}^{0}$ & 49468.05 & -10 & $\therefore 4$ \\
\hline 3056.73 & $a^{5} G_{2}-t^{5} F_{2}^{0}$ & 49346.30 & -1.49 & 17 \\
\hline $3061.59: \therefore$ & $a^{5} G_{2}-t^{5} F_{2}^{0}$ & 49346.30 & -0.53 & 15 \\
\hline 3064.28 & $a^{5} G_{b}-x^{5} G_{b}^{0}$ & 49408.54 & +0.51 & 27 \\
\hline 3070.90 & $a^{5} G_{4}-x^{5} G_{5}^{0}$ & 49301.98 & -0.06 & 27 \\
\hline $3074.37:: !$ & $a^{5} G_{5}-x^{5} G_{5}^{0}$ & 49301.98 & +0.60 & 18 \\
\hline $3080.40 \%$ & $a^{5} G_{3}-x^{5} G_{4}^{0}$ & $49 \quad 146.69$ & -0.11 & 27 \\
\hline 3085.62 & $a^{5} G_{4}-x^{5} G_{4}^{0}$ & $49 \quad 146.69$ & +0.48 & 27 \\
\hline $3089.14:$ & $a^{5} G_{5}-x^{5} G_{4}^{0}$ & $49 \quad 146.69$ & -0.50 & 27 \\
\hline 3101.34 & $a^{5} G_{z}-x^{5} G_{2}^{0}$ & $48 \quad 875.84$ & +0.68 & 28 \\
\hline 3112.12 & $a^{\top} S_{3}-z^{7} D_{4}^{0}$ & 32123.16 & -1.07 & 10 \\
\hline 3132.59 & $a^{7} S_{3}-y^{7} P_{4}^{0}$ & 31913.24 & +0.34 & 9 \\
\hline 3147.36 & $a^{5} G_{6}-u^{5} F^{0}$ & $48 \quad 547.44$ & -0.08 & 23 \\
\hline 3158.16 & $a^{7} S_{3}-z^{7} D_{3}^{0}$ & 31654.80 & -0.33 & 12 \\
\hline 3170.34 & $a^{7} S_{3}-y^{7} P^{0}$ & 31533.27 & +0.11 & 12 \\
\hline 3176.80 & $a^{5} S_{2}-w^{5} D_{2}^{0}$ & $42 \quad 237.41$ & -1.81 & 19 \\
\hline $3183.04 \cdots$ & $a^{5} G_{5}-u^{5} F_{4}^{0}$ & $48 \quad 192.05$ & -0.69 & 16 \\
\hline 3183.26 & $\mathrm{a}^{5} \mathrm{G}_{5}-y^{3} \mathrm{H}^{0}$ & $48 \quad 189.22$ & -0.06 & 22 \\
\hline $3187.69:$ & $a^{5} P_{3}-z^{5} F_{4}^{0}$ & 49590.83 & -0.53 & 19 \\
\hline 3193.97 & $a^{7} S_{3}-y^{7} P_{2}^{0}$ & 31299.95 & +0.05 & 13 \\
\hline $3200.22 x$ & $a^{5} p_{3}-t^{5} F_{3}^{0}$ & 49468.05 & -1.04 & 25 \\
\hline 3205.21 & $a^{5} D_{0}-w^{5} D_{i}^{0}$ & $42 \quad 156.14$ & -0.45 & 20 \\
\hline 3205.89 & $a^{5} D_{4}-x^{5} F_{4}^{0}$ & 43529.85 & +0.15 & 25 \\
\hline 3208.83 & $\mathrm{a}^{7} \mathrm{~S}_{3}-\mathrm{z}^{7} \mathrm{D}_{2}^{0}$ & $31 \quad 155.04$ & -0.64 & 11 \\
\hline $3215.07 n$ & $a^{5} D_{1}-w^{5} D_{2}$ & $42 \quad 237.41$ & -0.34 & 13 \\
\hline 3228.21 & $a^{5} D_{2}-w^{5} D_{3}^{0}$ & $42 \quad 422.40$ & -0.31 & 18 \\
\hline 3229.80 & $a^{5} D_{4}-x^{5} F_{5}^{0}$ & $43 \quad 299.08$ & -0.24 & 27 \\
\hline 3233.21 & $a^{5} G_{5}-y^{5} G_{6}^{0}$ & 47704.68 & +0.32 & 20 \\
\hline 3247.61 & $a^{5} D_{2}-w^{5} D_{2}^{0}$ & $42 \quad 237.41$ & -0.72 & 16 \\
\hline $3270.90: 1$ & $a^{5} D_{3}-w^{5} D_{3}^{0}$ & $42 \quad 422.40$ & -0.34 & 19 \\
\hline 3289.01 & $a^{5} D_{4}-w^{5} D_{4}^{0}$ & 42741.82 & +0.09 & 35 \\
\hline
\end{tabular}


Table 2. (Contd)

\begin{tabular}{|c|c|c|c|c|}
\hline $\begin{array}{l}\text { Wavelength } \\
\text { ( })\end{array}$ & Transition & $\begin{array}{l}E_{m} \\
\left(\mathrm{~cm}^{-1}\right)\end{array}$ & $\lg (g f)$ & $\begin{array}{c}\text { Error } \\
(8)\end{array}$ \\
\hline $3290.82 \%$ & $a^{5} D_{3}-w^{5} D_{2}^{0}$ & $42 \quad 237.41$ & -0.23 & 14 \\
\hline $3303.35 \%$ & $a^{5} S_{2}-y^{5} F_{2}^{0}$ & 41032.01 & -1.62 & 22 \\
\hline $3305.56 \div 1$ & $a^{5} S_{2}-y^{5} F_{1}^{0}$ & 41011.74 & -1.09 & 5 \\
\hline 3325.67 & $a^{5} S_{2}-x^{5} D_{2}^{0}$ & $40 \quad 828.85$ & -0.97 & 10 \\
\hline $3327.31 \%$ & $a^{5} D_{0}-y^{5} F_{1}^{0}$ & 41011.74 & -0.86 & 14 \\
\hline $3336.51 \%$ & $a^{5} P_{3}-u^{5} F_{4}^{0}$ & $48 \quad 192.05$ & -1.62 & 17 \\
\hline 3340.15 & $a^{5} S_{2}-x^{5} D_{1}^{0}$ & 40698.46 & -1.29 & 10 \\
\hline $3344.73 \% 1$ & $a^{5} D_{1}-y^{5} F_{2}^{0}$ & 41032.01 & -0.25 & 21 \\
\hline $3347.00 \%$ & $a^{5} D_{1}-Y^{5} F_{1}^{0}$ & 41011.74 & -0.87 & 24 \\
\hline 3358.12 & $a^{5} D_{2}-y^{s} F_{3}^{0}$ & 41224.41 & +0.03 & 12 \\
\hline 3362.36 & $a^{5} D_{0}-x^{5} D_{1}^{p}$ & 40698.46 & -1.18 & 17 \\
\hline 3363.77 & $a^{5} S_{2}-x^{5} D_{3}^{0}$ & $40 \quad 488.35$ & -0.56 & 14 \\
\hline $3379.97 \%$ & $a^{5} D_{2}-Y^{5} F_{2}^{0}$ & 41032.01 & -0.39 & 20 \\
\hline $3382.29 \%$ & $a^{5} D_{2}-y^{5} F_{1}^{9}$ & 41011.74 & -1.61 & 21 \\
\hline 3382.48 & $a^{5} D_{1}-x^{5} D_{1}^{p}$ & 40498.46 & -0.78 & 28 \\
\hline 3384.61 & $\mathrm{a}^{5} \mathrm{D}_{3}-\mathrm{Y}^{5} \mathrm{~F}_{4}^{0}$ & 41395.54 & +0.12 & 22 \\
\hline $3389.69 \%$ & $b^{5} D_{4}-t^{5} F_{5}^{9}$ & 49650.68 & -1.62 & 23 \\
\hline 3397.68 & $a^{5} D_{1}-z^{3} D_{2}^{0}$ & 40.566 .27 & -1.47 & 15 \\
\hline 3404.34 & $a^{5} D_{3}-Y^{5} F_{3}^{0}$ & 41224.41 & -0.46 & 16 \\
\hline $3425.20 \%$ & $b^{5} D_{2}-t^{5} E_{3}^{0}$ & $49 \quad 468.05$ & -0.79 & 25 \\
\hline $3426.79:$ & $a^{5} D_{3}-y^{5} F_{2}^{0}$ & 41032.01 & -1.28 & 23 \\
\hline $3433.38 \%$ & $b^{5} D_{3}-t^{5} F_{3}^{0}$ & 49468.05 & -1.05 & 25 \\
\hline 3434.78 & $a^{5} D_{3}-z^{3} D_{3}^{0}$ & 40964.11 & -0.45 & 19 \\
\hline $3439.56 \% 1$ & $b^{5} D_{2}-t^{5} F_{2}^{2}$ & 49346.30 & -1.19 & 16 \\
\hline 3447.12 & $a^{5} D_{4}-y^{5} F_{5}^{0}$ & 41347.71 & +0.50 & 20 \\
\hline 3456.39 & $a^{7} S_{3}-z^{3} P_{3}^{0}$ & 28923.66 & -1.57 & 16 \\
\hline $3490.26 \%$ & $a^{3} G_{3}-t^{5} F_{4}^{0}$ & $49 \quad 590.83$ & -0.53 & 18 \\
\hline $3521.42 \%$ & $b^{5} D_{4}-U^{5} F_{5}^{0}$ & $48 \quad 547.44$ & +0.42 & 24 \\
\hline $3566.06 \%$ & $b^{5} D_{4}-u^{5} F_{4}^{0}$ & $48 \quad 192.05$ & -0.73 & 17 \\
\hline 3581.88 & $a^{5} G_{5}-y^{5} H_{6}^{0}$ & $44 \quad 694.93$ & +0.16 & 19 \\
\hline $3590.74: 1$ & $b^{5} D_{3}-u^{5} F_{4}^{0}$ & $48 \quad 192.05$ & -0.81 & 17 \\
\hline 3614.31 & $a^{5} G_{5}-y^{5} H_{5}^{0}$ & $44 \quad 444.51$ & +0.40 & 55 \\
\hline 3624.45 & $a^{5} G_{4}-z^{5} I_{5}^{0}$ & $44 \quad 330.23$ & +0.22 & 27 \\
\hline $3697.43 \%$ & $a^{3} G_{4}-u^{5} F_{4}^{0}$ & $48 \quad 192.05$ & -1.77 & 17 \\
\hline $3723.50 \%$ & $a^{3} G_{s}-u^{5} F_{4}^{0}$ & $48 \quad 192.05$ & -1.36 & 17 \\
\hline 3798.26 & $a^{7} S_{3}-z^{7} P_{4}^{0}$ & 26320.38 & +0.12 & 8 \\
\hline $3825.32 \%$ & $a^{3} F_{4}-t^{5} F_{5}^{0}$ & 49650.68 & -0.62 & 22 \\
\hline 3826.69 & $a^{5} D_{2}-z^{5} D_{2}^{2}$ & 37579.23 & -0.85 & 20 \\
\hline $3854.91 \%$ & $a^{3} F_{2}-t^{5} F_{3}^{0}$ & 49468.05 & -1.26 & 25 \\
\hline $3856.52 \%$ & $a^{3} F_{3}-t^{5} F_{4}^{0}$ & $49 \quad 590.83$ & -0.79 & 19 \\
\hline 3864.11 & $a^{7} S_{3}-z^{7} P^{0}$ & 25871.86 & +0.01 & 11 \\
\hline $3885.51 \%$ & $a^{5} G_{3}-w^{5} D_{3}^{0}$ & $42 \quad 422.41$ & -1.75 & 18 \\
\hline
\end{tabular}


Table 2. (Contd)

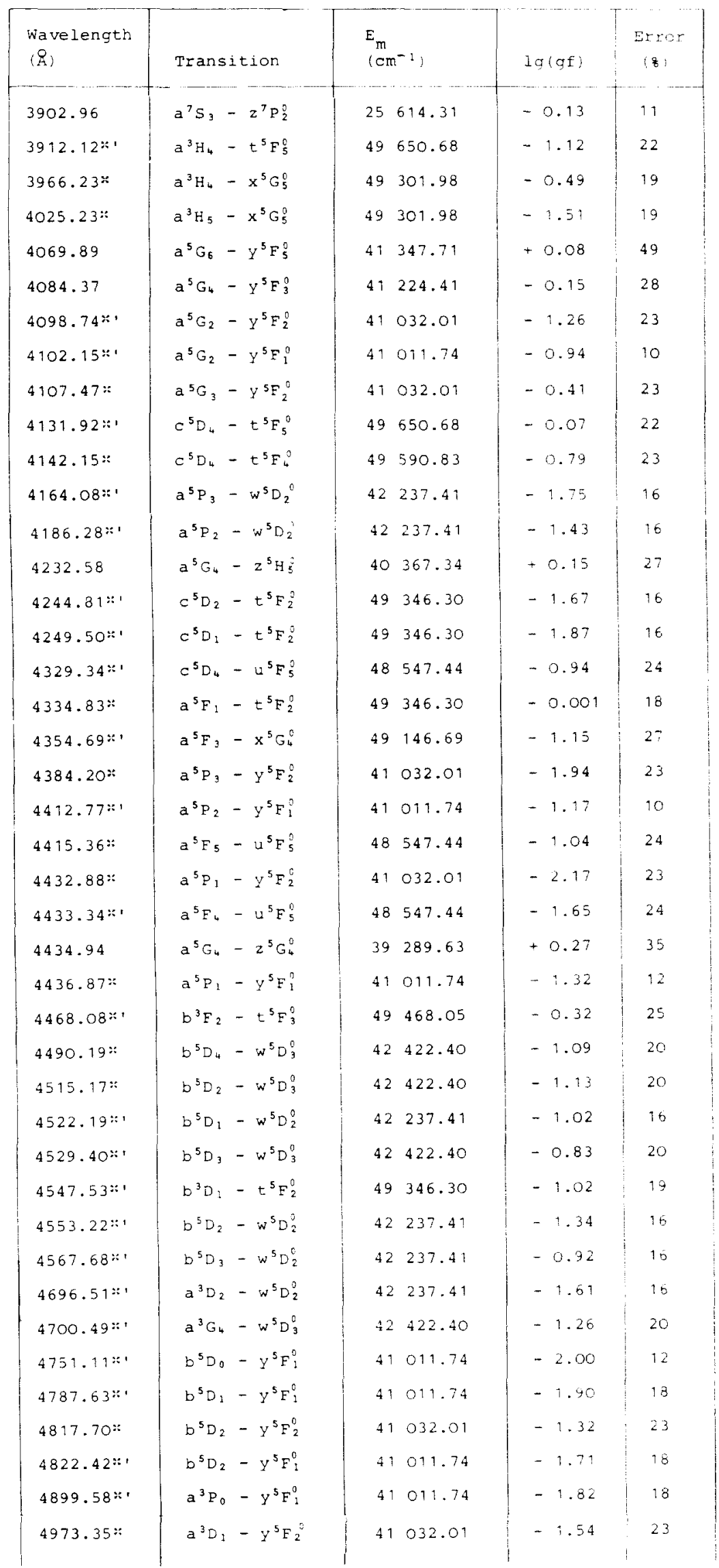


Table 2. (Contd)

\begin{tabular}{|l|l|l|l|c|}
\hline $\begin{array}{l}\text { Wavelength } \\
(\AA)\end{array}$ & Transition & $\begin{array}{l}E_{\mathrm{m}} \\
\left(\mathrm{cm}^{-1}\right)\end{array}$ & $\lg (g f)$ & $\begin{array}{c}\text { Error } \\
(8)\end{array}$ \\
\hline $4977.69 * 1$ & $\mathrm{a}^{3} \mathrm{G}_{3}-\mathrm{y}^{5} \mathrm{~F}_{2}^{0}$ & 41032.01 & -1.73 & 23 \\
$4983.45: 1$ & $\mathrm{a}^{3} \mathrm{D}_{2}-\mathrm{y}^{5} \mathrm{~F}_{2}^{0}$ & 41011.74 & -1.85 & 18 \\
$5114.69 *$ & $\mathrm{a}^{3} \mathrm{P}_{2}-\mathrm{w}^{5} \mathrm{D}_{3}^{0}$ & 42422.40 & -2.45 & 20 \\
5506.50 & $\mathrm{a}^{5} \mathrm{~S}_{2}-\mathrm{z}^{5} \mathrm{P}_{3}^{0}$ & 28923.66 & +0.047 & 11 \\
5533.03 & $\mathrm{a}^{5} \mathrm{~S}_{2}-\mathrm{z}^{5} \mathrm{P}_{2}^{0}$ & 28836.60 & -0.103 & 5 \\
5570.43 & $\mathrm{a}^{5} \mathrm{~S}_{2}-\mathrm{z}^{5} \mathrm{P}_{1}^{0}$ & 28715.29 & -0.413 & 18 \\
\hline
\end{tabular}

With the highly resolved Fourier spectrum, we were also able to correct wavelengths of molybdenum lines (indicated by a prime in Tables 2 and 3). The corrections are of the order $\pm 5 \mathrm{~m} \AA$ in relation to the wavelengths calculated from the energy values given by Moore. ${ }^{15}$ We have checked our laboratory spectra for blends and misidentifications. Distorted lines have been rejected. Finally, we measured relative $f$-values from this spectrum. These transitions are marked by an asterisk in Tables 2 and 3.

Table 3. Relative oscillator strengths of Mo(II) lines: $\left({ }^{*}\right)$, determined from a Fourier spectrum; $\left({ }^{\prime}\right)$, wavelength corrected.

\begin{tabular}{|c|c|c|c|c|}
\hline $\begin{array}{l}\text { Wavelength } \\
(\AA)\end{array}$ & Transition & $E_{\mathrm{m}}\left(\mathrm{cm}^{-1}\right)$ & $\lg (g f) \mathrm{rel}$ & $\begin{array}{c}\text { Error } \\
(8)\end{array}$ \\
\hline 2538.44 & $a^{6} D_{9 / 2}-z^{4} F_{9 / 2}^{0}$ & 52843.10 & +0.09 & 11 \\
\hline 2542.67 & $a^{6} D_{7 / 2}-z^{4} F_{7 / 2}^{0}$ & $52 \quad 217.30$ & -0.3 & 20 \\
\hline 2542.79 & $a^{6} D_{5 / 2}-z^{4} F_{5 / 2}^{0}$ & 51732.39 & -0.61 & 32 \\
\hline 2593.71 & $a^{6} D_{3 / 2}-z^{6} D_{5 / 2}^{0}$ & $50 \quad 577.36$ & -0.45 & 8 \\
\hline 2602.80 & $a^{6} D_{3 / 2}-z^{6} D_{3 / 2}^{0}$ & 50192.00 & -0.56 & 6 \\
\hline 2619.35 & $a^{6} D_{1 / 2}-z^{6} D_{1 / 2}^{0}$ & 49949.45 & -0.80 & 12 \\
\hline 2636.67 & $a^{6} D_{3 / 2}-2^{6} D_{1 / 2}^{0}$ & 49949.45 & -0.3 & 16 \\
\hline 2638.76 & $a^{6} D_{5 / 2}-z^{6} D_{7 / 2}^{0}$ & $50 \quad 302.54$ & +0.15 & 11 \\
\hline 2644.35 & $a^{6} D_{, / 2}-z^{6} D_{9 / 2}^{0}$ & 50705.52 & +0.18 & 14 \\
\hline 2646.49 & $a^{6} D_{5 / 2}-z^{6} D_{3 / 2}^{0}$ & 50192.00 & -0.2 & 6 \\
\hline 2653.35 & $a^{6} D_{7 / 2}-z^{6} D_{5 / 2}^{0}$ & $50 \quad 577.36$ & -0.07 & 9 \\
\hline 2660.58 & $a^{6} D_{3 / 2}-z^{6} P_{5 / 2}^{0}$ & 49608.74 & 0 & - \\
\hline 2672.84 & $a^{6} D_{7 / 2}-z^{6} D_{7 / 2}^{0}$ & $50 \quad 302.54$ & +0.21 & 14 \\
\hline 2683.23 & $a^{6} D_{1 / 2}-z^{6} P_{3 / 2}^{0}$ & 49040.82 & -0.05 & 8 \\
\hline 2684.14 & $a^{6} D_{9 / 2}-2^{6} D_{9 / 2}^{0}$ & 50705.22 & +0.41 & 10 \\
\hline 2687.99 & $a^{6} D_{5 / 2}-z^{6} p_{5 / 2}^{0}$ & 49608.74 & -0.03 & 5 \\
\hline 2701.41 & $a^{6} D_{3 / 2}-z^{6} P_{3 / 2}^{0}$ & 49040.82 & -0.15 & 7 \\
\hline 2713.50 & $a^{6} D_{9 / 2}-z^{6} D_{7 / 2}^{0}$ & $50 \quad 302.54$ & -0.56 & 28 \\
\hline 2729.68 & $a^{6} D_{5 / 2}-z^{6} P_{3 / 2}^{0}$ & 49040.82 & -0.59 & 18 \\
\hline 2732.87 & $a^{6} D_{7 / 2}-2^{6} P_{7 / 2}^{0}$ & $49 \quad 481.04$ & -0.38 & 10 \\
\hline 2775.39 & $a^{6} D_{9 / 2}-z^{6} P_{7 / 2}^{0}$ & $49 \quad 481.04$ & +0.52 & 11 \\
\hline 2784.98 & $a^{4} D_{7 / 2}-z^{4} F_{9 / 2}^{0}$ & 52843.10 & +0.24 & 17 \\
\hline 2807.75 & $a^{6} D_{5 / 2}-z^{4} P_{3 / 2}^{0}$ & 48022.45 & -0.29 & 14 \\
\hline
\end{tabular}


Table 3. (Contd)

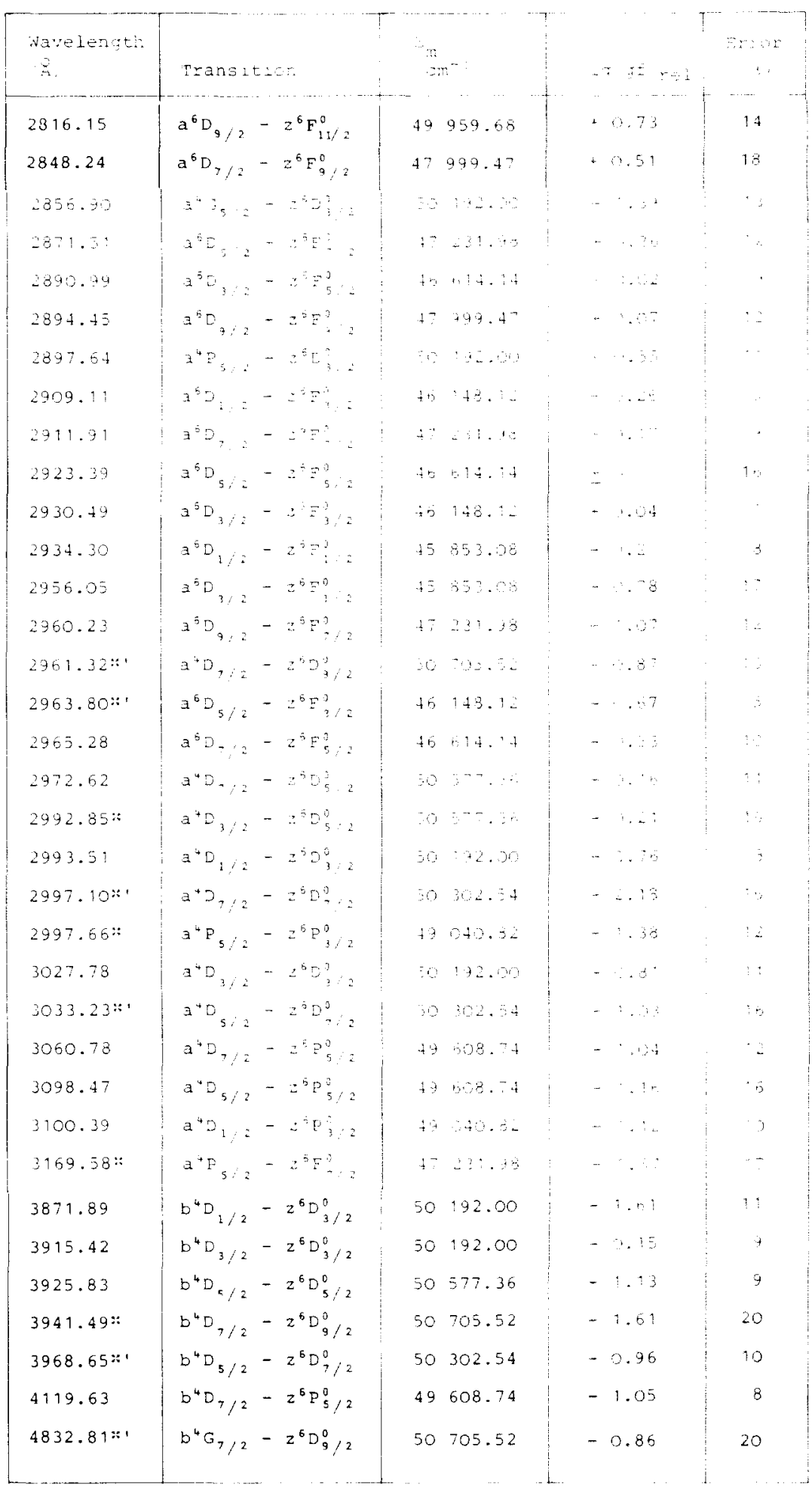

We used the fourth lifetime of Table 1 for an independent second absolute calibration of one of the three sets of relative $f$-values and compared that result with the first one. The consistency between both results is within $10 \%$.

A comparison of our Mo(I) $f$-values with published data is shown in Fig. 1. Absolute data were published by Corliss and Bozman. ${ }^{16}$ Their data were obtained from simplified emission measurements on free-burning arcs operated in air. 
The method has been critically analysed by several authors ${ }^{17,18}$ and was found to be prone to error. As can be seen from Fig. 1, the deviations from our data reach a factor of 100 , especially for small $f$-values. In our opinion, the reason for the large discrepancies is the absence of LTE in their plasma light source together with an inadequate temperature determination. Also, the absolute scale seems too high by about 0.5 dex. Nevertheless, the comprehensive data published by Corliss and Bozman ${ }^{16}$ are still a valuable aid for orientation when one tries to measure oscillator strengths.

Mishakov and Fogel ${ }^{19}$ used a pulsed discharge in order to excite $\mathrm{MoCl}_{5}$. They applied the hook technique to obtain relative $f$-values. We have linked their set to ours via the line $3170 \AA$. As may be seen in Fig. 1, the agreement is very good.

Dickerman and Deuel ${ }^{20}$ have measured a few relative molybdenum $f$-values of which we have only two in common. We have not included these two data in our comparison.

Kwiatkowski et al. ${ }^{13}$ have converted their measured Mo(I) lifetimes into absolute oscillator strengths under the assumption that the resonance transition is dominant. Their results agree very well with ours. The deviations are normally smaller than $10 \%$. We found

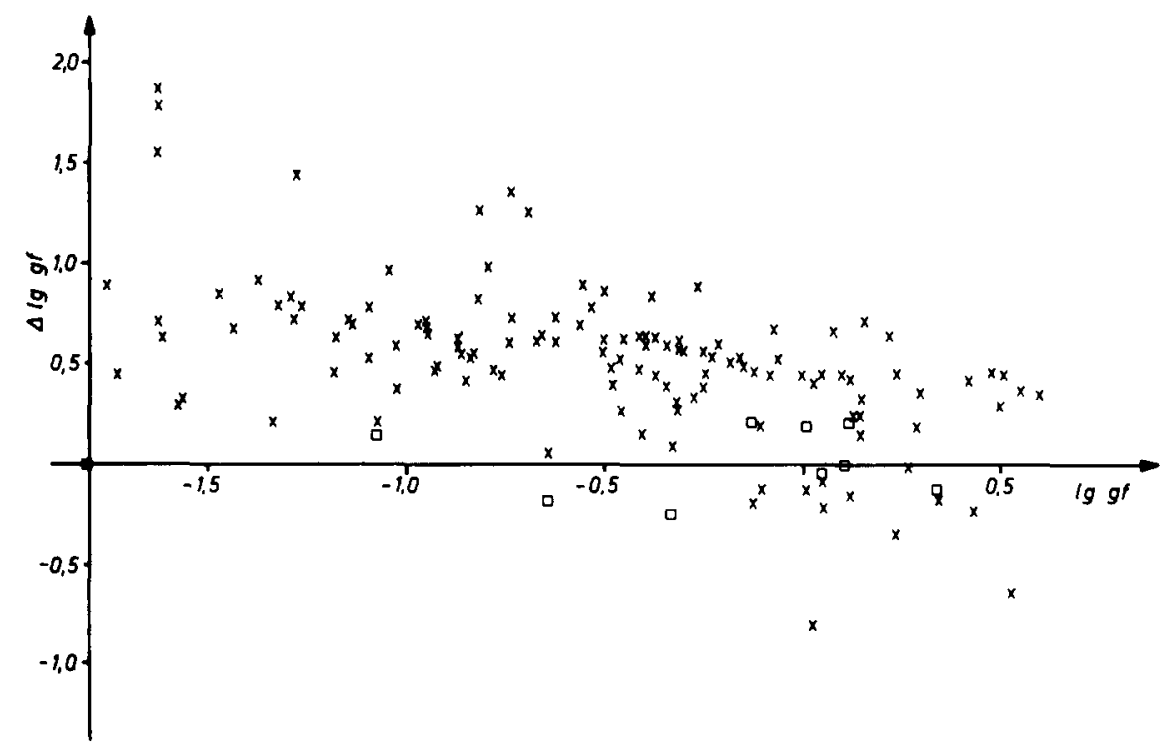

Fig. 1. Comparison of the present Mo(I) oscillator strengths with other results: $\times$, Corliss and Bozman; ${ }^{16} \square$, Mishakov and Fogel. ${ }^{19}$

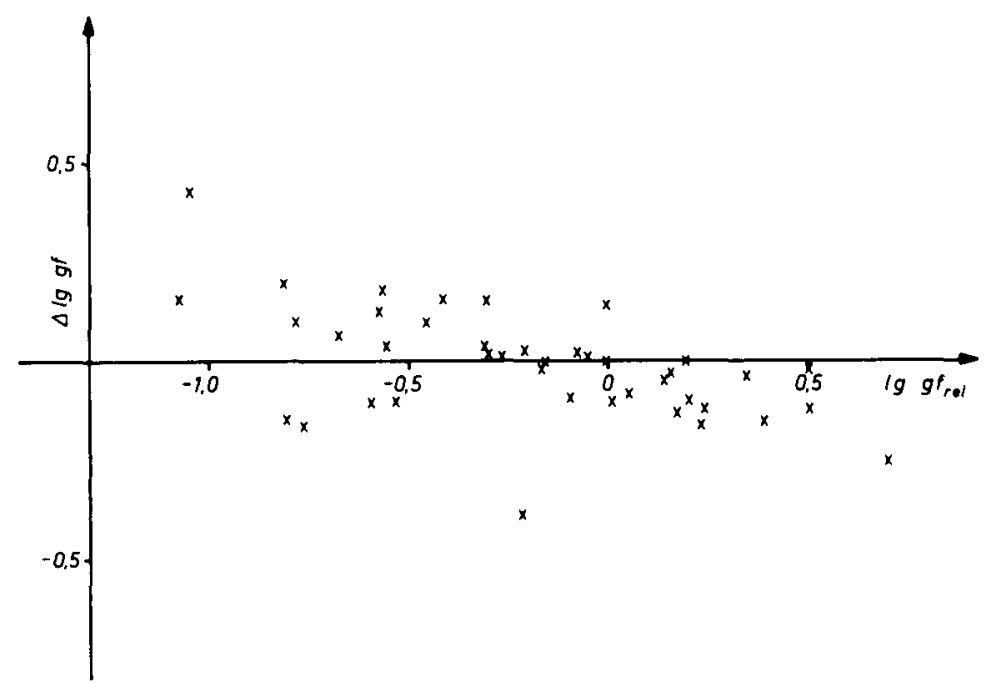

Fig. 2. Comparison of the present relative $\mathrm{Mo}$ (II) oscillator strengths with the results of Corliss and Bozman. ${ }^{16}$ 
deviations larger than $10 \%$ for only two lines. This result corroborates both the lifetime measurements as well as our data.

The $58 \mathrm{Mo}$ (II) $f$-values are listed in Table 3. Because of missing lifetimes, the values are only on a relative scale. The quoted errors have been determined in the same way as for the Mo(I) data. For comparison, we only found the data published by Corliss and Bozman. ${ }^{16}$ Both scales are linked together via the line $2660 \AA$. As can be seen from Fig. 2 , the scatter is obviously smaller than for the $\operatorname{Mo}(\mathrm{I})$ data, but there is again increased deviation towards smaller $f$-values.

\section{CONCLUSION}

Molybdenum is a highly refractory metal with very complex spectra. We consider the absolute $\operatorname{Mo}(\mathrm{I}) f$-values to have satisfactory accuracy. Neither the assumption of LTE nor temperature measurements are required here. Comparisons with the few literature data show differences by factors of up to 100 . For Mo(II), lifetimes are not yet available. Therefore only relative $f$-values are given.

Acknowledgements--This work was supported by Deutsche Forschungsgemeinschaft. We would like to thank P. Zimmermann and his group for communicating to us the Mo(I) lifetimes. We also thank E. Schulz-Gulde for a critical reading of the manuscript.

\section{REFERENCES}

1. W. L. Wiese, Methods of Experimental Physics, Vol. 7A, p. 117. Academic Press, New York (1968)

2. R. Ladenburg, Rev. Mod. Phys. 5, 243 (1933).

3. M. Kock and M. Kühne, J. Phys. B10, 3421 (1977).

4. M. Kühne, K. Danzmann and M. Kock, Astron. Astrophys. 64, 111 (1978).

5. B. L. Cardon, P. L. Smith. and W. Whaling, Phys. Rev. A20, 2411 (1979).

6. K. Danzmann and M. Kock. J. Phys. B13, 2051 (1980).

7. H. A. Bachor and M. Kock, J. Phys. B13, 2497 (1980)

8. D. Einfeld and D. Stuck, Z. Naturf. 33a, 502 (1978).

9. H. Magdeburg and U. Schley, Z. Angen. Phys. 20, 465 (1966).

10. D. Einfeld, K. Grützmacher, and D. Stuck, Z. Naturf. 34a, 233 (1979).

11. K. Danzmann and M. Kock, JOSA 72, 1556 (1982).

12. P. Zimmermann, Private communication.

13. M. Kwiatkowski, G. Micali, K. Werner, and P. Zimmermann, Phys. Letl. 85A, 273 (1981).

14. R. L. Kurucz and E. Peytreman, "A Table of Semiempirical gf Values", SAO Special Rep. 362. Smithsonian Astrophysical Observatory, Cambridge, Mass. (1975).

15. CH. Moore, "Atomic Energy Levels", Vol. III. NBS Circular 467. Washington (1971).

16. C. H. Corliss and W. Bozman, NBS Monograph 53, Washington (1962)

17. R. A. Bell and W. L. Upson, Astrophys. Lett. 9, 109 (1971).

18. B. Warner and C. R. Cowley, JQSRT 7, 751 (1967).

19. V. G. Mishakov and N. J. Fogel, Opt. Spectrosc. 42, 1193 (1976)

20. P. J. Dickerman and R. W. Deuel, JQSRT 4, 807 (1964) 